\title{
Retrospective Analysis of Survival in Patients with Brain Metastases from an Unknown Primary Tumor
}

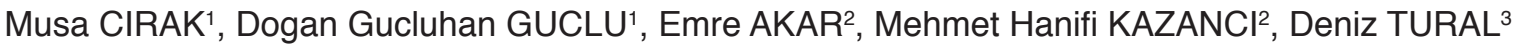 \\ ${ }^{1}$ University of Health Sciences Bakirkoy Dr. Sadi Konuk Training and Research Hospital, Department of Neurosurgery, Istanbul, Turkey \\ ${ }^{2}$ University of Health Sciences Bakirkoy Dr. Sadi Konuk Training and Research Hospital, Department of Internal Medicine, Istanbul, Turkey \\ ${ }^{3}$ University of Health Sciences Bakirkoy Dr. Sadi Konuk Training and Research Hospital, Departement of Medical Oncology, Istanbul, Turkey
}

Corresponding author: Musa CIRAK musacirak@hotmail.com

\section{ABSTRACT}

AIM: To evaluate the patients with brain metastases from unknown primary (BMUP) cancers, and to analyze the prognostic factors and survival rates.

MATERIAL and METHODS: We conducted a retrospective study with 110 patients (mean age: 62.8 years [range 23-90], $\mathrm{n}=85-$ $77.3 \%$ male and $n=25-22.7 \%$ female) with BMUP cancers at the time of diagnosis, who presented to our outpatient oncology clinic between January 2015 and December 2019. We employed the following variables as significant prognostic factors for a prominent index of patients' survival: age, gender, Karnofsky performance score (KPS), number of metastatic lesions, primary site, and type of treatment were analyzed for their prognostic effects on survival outcomes. Patients' survival was evaluated from plotted KaplanMeier curves, and the log-rank test was used for univariate analysis.

RESULTS: The mean follow-up was 13 months (range 4-60 months). The means of survival after the diagnosis of brain metastasis was 18.7 months for the study group. Lung cancer was the most common primary tumor (74, 5\%). The KPS and number of lesions were found to have a prognostic effect on survival. Survival analysis showed no statistical significance with age and gender, primary site, type of treatment.

CONCLUSION: This study showed that KPS, and the number of lesions affect the survival outcomes but both the other variables. Therefore, BMUP cancer is indeed related to poor prognosis.

KEYWORDS: Brain metastases, Neoplasm, Survival analysis, Unknown primary, Prognosis

ABBREVIATIONS: BMUP: Brain metastases from unknown primary, KPS: Karnofsky performance score, NICE: National Institute for Health and Care Excellence, RTOG: The Radiation Therapy Oncology Group

\section{INTRODUCTION}

$\mathrm{B}$ rain metastases are the most common intracranial tumors with a 4-10 times higher prevalence rate than primary malignant brain cancers (9). The incidence rate of metastatic brain tumors has been increasing, mainly because brain metastasis has remained an indicator of poor prognosis with debilitating effects on many critical functions controlled by the organ and results in nearly always a fatal outcome in patients with solid cancers. Patients' survival is typically $<2$ years, and a few patients $(10 \%)$ would survive for $>5$ years (2).

Intracranial metastases are regarded as end-stage cancers related to significant morbidity and mortality (4). In the past, survival time of a patient with a metastatic cerebral tumor was approximately a month without treatment (10). Only palliative treatment was used, and personalized treatment modalities
Musa ÇIRAK (10): 0000-0002-0175-9655

Dogan Gucluhan GUCLU (D) : 0000-0002-9959-961X

Emre AKAR (1): 0000-0002-9834-7488
Mehmet Hanifi KAZANCI (1) : 0000-0001-9129-6393

Deniz TURAL (1) : 0000-0003-2144-6469 
had a limited potential to increase survival times (4). Lately, however, continuous developments in chemotherapy, notably in small cell lung cancer and breast cancer, have improved diagnostic methods that enable early detection and are expected to increase survival rates (7).

Although the primary tumor site is crucial for the choice of treatment, $15 \%$ of patients with metastatic intracranial tumors are diagnosed with the unknown primary site. Neurological symptoms of brain tumors can be the first sign of an undiagnosed metastatic disease (2). The National Institute for Health and Care Excellence (NICE) also highlighted the scarcity of data regarding brain metastases of unknown primary (6).

We aimed to retrospectively evaluate 110 patients with brain metastases of unknown primary cancer at the time of diagnosis and evaluate potential factors (age, gender, Karnofsky Performance Score, number of metastases, treatment type) that attribute to patients' survival time.

\section{MATERIAL and METHODS}

This retrospective study was conducted at Medical Oncology and Neurosurgery Departments of University of Health Sciences Bakirkoy Dr. Sadi Konuk Training and Research Hospital, between the dates of January 2015 and December 2019, enrolling 110 patients with brain metastases from unknown primary tumors at the time of diagnosis with consistent followed up and enrolled in the study. Exclusion criteria were as follows: patients with brain metastases from known primary sites and subjects without follow-up data.

The study was approved by our Institutional Review Board (Approval \#: 2020/195). Written informed consent was obtained from all participants.

Demographic and clinical characteristics were obtained from patients' medical records. We employed the following variables as significant prognostic factors for a prominent index of patients' survival: age, gender, mean survival time, performance status based on Karnofsky Performance Status (KPS; scores range: 0-100), number of metastatic lesions, the primary site that was detected in evaluations, and type of treatment (if metastasectomy performed or not). Overall survival was calculated from the date of diagnosis until death from any cause or last date of a patient known to be alive. For the detection of primary cancer, a routine diagnostic evaluation was done. Final identification of the primary tumor was based on histopathological examination of either cranial resection or tissue biopsy material from a radiologically-detected primary tumor.

Each of the parameter was divided as below 63 or over 63 years for age and male or female for gender. Patient performance which was assessed by Karnofsky score was divided as below 70 or over 70 score. Number of lesions were classified as single/two or over three for several lesions. Primary tumor site was classified as lung or another primary for primary site. Finally; treatment was classified as surgical or nonsurgical treatment of cranial metastases. The effect of each variable on survival was determined by univariate analysis.

\section{Statistical Analysis}

All analyses were carried out in Statistical Package for the Social Sciences (SPSS) 20.0 (IBM, USA). Numerical data were presented as a mean and standard deviation. Relative frequencies were described as percentages. Kaplan-Meier curves were plotted for evaluation of survival, and the log-rank test was used for univariate analysis. The level of significance was set at $\mathrm{p}<0.05$.

\section{RESULTS}

During the study period, 1423 patients with brain metastasis were admitted to the hospital. Among these, 127 (8.9\%) patients were diagnosed with unknown primary tumor. Hundred and ten ( $n=110,86.6 \%)$ out of 127 patients with unknown primary tumors had regular follow-ups and were enrolled in the study. Eighty-five ( $n=85,77.3 \%)$ out of 110 patients were male, and the remaining 25 (22.7\%) were female. The mean age of the patients was 62.8 years (range 23-90). Mean age of men $=63.8 \pm 10.3$ years, for women $=59.4 \pm$ 14.9 years. The primary cancer type was determined with histopathological examination of either resection material of cerebral metastasis $(n=14,12.7 \%)$ or tissue biopsy material of radiologically-detected primary tumor $(n=96,87 \%)$. Fourteen patients $(n=14)$ were treated with cranial metastasectomy and radiotherapy; 96 patients only received cranial radiotherapy as a local treatment. All patients received palliative systemic chemotherapy based on primary tumor pathology.

After the diagnostic examinations, the most common primary site for cancer was found to be the lung $(n=82,74.5 \%)$, followed by breast $(n=4,3.6 \%)$, kidney $(n=4,3.6 \%)$, colon $(n=3,2.7 \%)$, gastric $(n=3,2.7 \%)$, rectum $(n=3,2.7 \%)$, and laryngeal $(n=3,2.7 \%)$, and melanoma $(n=3,2.7 \%)$. There was one case each of pancreas, uterus, bladder, esophageal, and biliary tract $(n=1,0.9 \%)$.

Fifty-four ( $n=54,49 \%$ ) out of 110 patients were below 63 years, and the remaining $56(51 \%)$ subjects were over 63 years. Regarding functional status, 70 patients were assigned a KPS $\geq 70(n=70,63.6 \%)$, while 40 patients had a KPS $<70$ $(n=40,36.4 \%)$. Fifty-eight $(n=58,52.7 \%)$ out of 110 patients had one or two metastatic lesions, and the remaining 52 $(47.2 \%)$ subjects had $\geq 3$ lesions. In 14 (12.7\%) patients metastasectomy was performed, and in 96 (87.2\%) patients, no surgical treatment was performed (Table I).

The death occurred in 28 patients (25\%). Lung tumors accounted for most deaths $(n=23)$, followed by melanoma $(n=2)$. One gastric tumor, one colon cancer, and one bladder cancer were responsible for the other three deaths.

The mean follow-up was 13 months (range 4-60 months). Mean survival after diagnosis was 18.7 months (range 16.3521.17) (17.7 months for men and 19 months for women). From the factors evaluated, KPS and the number of metastatic lesions were found to have a prognostic effect on survival time. Mean survival time, according to KPS, was 22.1 months for $K P S \geq 70$ and 15.4 months for $K P S<70 \quad(p=0.006)$ (Figure 1). Mean survival time according to the number of metastatic 
Cirak M. et al: Brain Metastases from Unknown Primary Tumors

Table I: Univariate Analysis Characteristics on Survival in Patients' with Brain Metastases of an Unknown Site of a Primary Tumor

\begin{tabular}{|c|c|c|c|c|}
\hline Parameter & Subgroup & Number of patients $n(\%)$ & Survival (months) & $\mathbf{p}$ \\
\hline \multirow{2}{*}{ Age } & $<63$ & $54(49)$ & 17.8 & \multirow{2}{*}{0.37} \\
\hline & $\geq 63$ & $56(51)$ & 20 & \\
\hline \multirow{2}{*}{ Gender } & Female & $25(22.7)$ & 17.7 & \multirow{2}{*}{0.184} \\
\hline & Male & $85(77.3)$ & 19 & \\
\hline \multirow{2}{*}{$\mathrm{KPS}^{\mathrm{a}}$} & $<70$ & $40(36.3)$ & 15.4 & \multirow{2}{*}{0.006} \\
\hline & $\geq 70$ & $70(63.6)$ & 22.1 & \\
\hline \multirow{2}{*}{ Number of lesions } & $<3$ & $58(52.7)$ & 21.7 & \multirow{2}{*}{0.002} \\
\hline & $\geq 3$ & $52(47.2)$ & 14.5 & \\
\hline \multirow{2}{*}{ Primary Tumor site } & Lung & $82(74.5)$ & 17.7 & \multirow{2}{*}{0.24} \\
\hline & Other & $28(25.4)$ & 21.4 & \\
\hline \multirow{2}{*}{$\begin{array}{l}\text { Surgical treatment } \\
\text { (metastasectomy) }\end{array}$} & Yes & $14(12.7)$ & 17.5 & \multirow{2}{*}{0.58} \\
\hline & No & 96 (87.2) & 18.9 & \\
\hline
\end{tabular}

a: KPS: Karnofsky Performance Status.

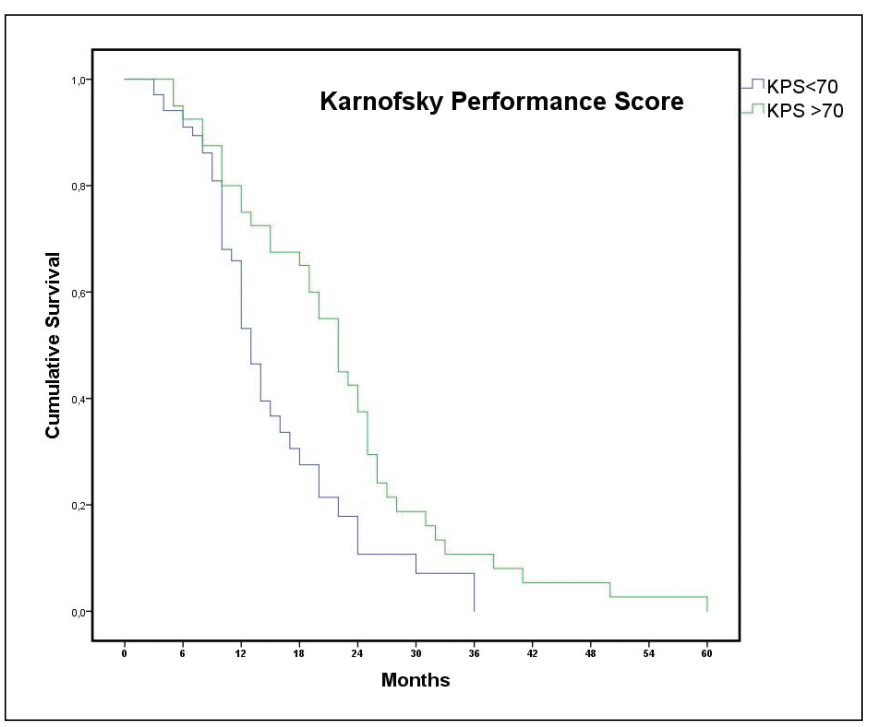

Figure 1: Kaplan-Meier curves of survival according to the Karnofsky Performance Score.

brain lesions was 21.7 months for $<3$ lesions and 14.5 months for $\geq 3$ lesions $(p=0.002)$ (Figure 2).

Mean survival time did not differ among the age groups below or over 63 years $(p=0.373)$. There was also no significant difference in mean survival time according to gender $(p=0.184)$, tumor site $(p=0.245)$, and type of treatment $(p=0.582)$.

\section{DISCUSSION}

Patients $(n=110)$ with brain metastasis of unknown primary location at diagnosis were retrospectively evaluated in this

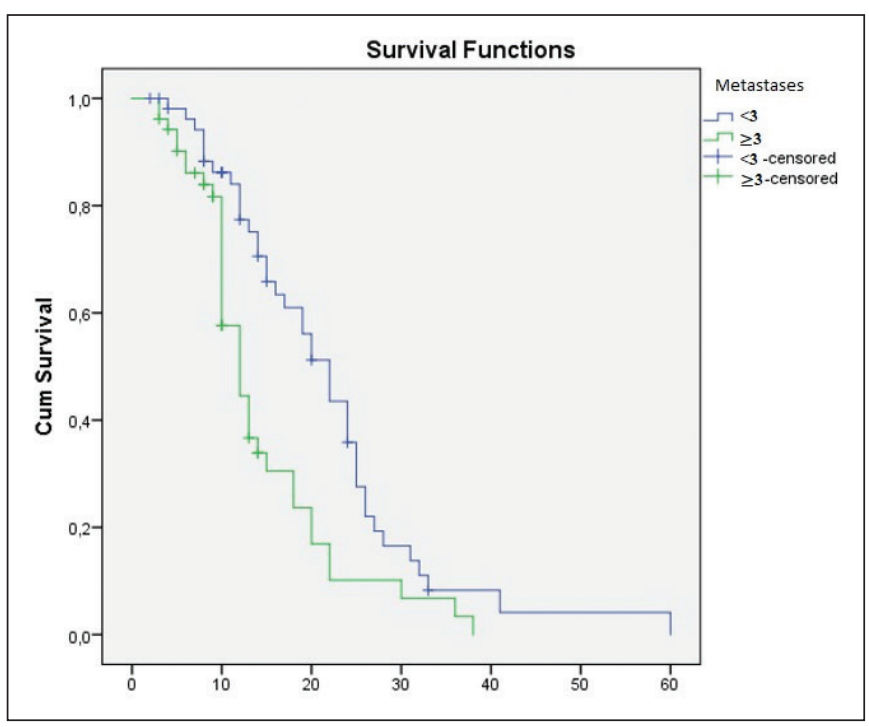

Figure 2: Kaplan-Meier curves of survival according to the number of brain metastases.

study to determine the prognostic factors affecting these patients' survival time. The mean survival time was 18.7 months. Demographic data (age, gender), KPS, number of metastatic lesions, the primary tumor type, and type of treatment were evaluated in univariate analysis. From the factors examined, only KPS below 70 and cerebral metastases number at least or more than three correlated with decreased survival rates.

The frequency of metastatic cerebral tumors is increased due to improved systemic disease control, advanced imaging modalities, and increased use of routine screening for staging. 
Cerebral metastases constitute $10-35 \%$ of all cerebral metastatic lesions (9). These metastatic tumors had different characteristics of complicating treatment, prognosis, and treatment responses. Nevertheless, cerebral metastases, typically, are considered to be related to poor prognosis (8). Treatment options might differ with the number of metastases, cerebral location, patients' performance status, and primary cancer type. The use of steroids is the first step of treatment after diagnosis. Surgery or radiosurgery might be prioritized in patients with a single metastatic lesion. In the case of multiple metastatic tumors $(>3)$, whole-brain radiotherapy with or without a combination of chemotherapy is generally preferred. The efficacy of systemic chemotherapeutics is limited and might be suggested in the selected cases $(2,9)$.

Overall, most of the metastatic lesions to the brain were primed from lung, breast, and melanoma. Other cancers that tended to migrate to the brain included colorectal carcinoma, renal cancers, thyroid, and gastrointestinal malignancies (4) Majority of the primary tumors identified in our study were mostly lung, breast, and renal carcinomas, consistent with reports in the literature. The lung was the major primary location of the metastatic disease $(74.5 \%)$; however, breast cancer constituted only a small part (3.6\%). In previous publications, the rate of breast cancer was reported to be higher and was attributable to the heterogeneity of our study population $(11,12)$.

Brain metastasis from unknown primary cancers is a relatively rare phenomenon; therefore, only a limited number of cases have been reported. In 2018, The National Institute for Health and Care Excellence reported a series of 298 patients with brain metastasis in which 55 subjects categorized as brain metastasis from unknown primary (6). In another study, Rotta et al. reported 71 subjects (11). Both studies highlighted the scarcity of data regarding brain metastases of unknown primary. However, our report presents one of the most extensive patient series of BUMP cancer. Otherwise, reported data focused mainly on brain metastasis with known primary tumor origin. Abroad spectrum study by Gaspar et al. involving 1200 patients from three different brain metastasis trials, showed that subjects treated with radiation therapy, Gaspar et al. reported that the most favorable prognosis was observed in patients who were younger than 65 years old, who had a higher KPS than 70, who had the primary disease under control and who had only isolated brain metastasis (5). If these all four factors were present, patients had a $52 \%$ probability of survival for at least 200 days. Similarly, studies with 2000 patients by Sperduto et al. reported four prognostic factors regarding improved survival in brain metastases. These four factors included patients' performance status (KPS $\geq 70$ ), age $(<60)$, the number of brain metastases $(<3)$, and metastases limited to the brain. A graded prognostic assessment classification is established with these factors (13).

In this study cohort, age was not shown to have a statistically significant prognostic effect on survival. This finding is consistent a previous similarly designed studies (11), but not with radiation therapy oncology group (RTOG) classification (13). However, high KPS and a low number of lesions had a statistically significant prognostic effect on survival in this study despite previous publications (11). Finally, in a study with an enrollment of 71 subjects, Rotta et al. reported that only gender affects survival, without any noticeable effects of age, histological type, KPS, location of cerebral metastases, and treatment type on survival (11). Similarly our results showed that surgically (metastasectomy) treated patients had similar outcomes and survival rates with those not treated with surgery. Although the sample size is unbalanced, and the metastasectomy group had very few patients $(n=14,12.7 \%)$, These findings suggested that local treatment of cerebral metastases does not significantly change the disease course. Gough et al. reported the results of 298 subjects with brain metastases (343-82\% patients with known primary and 55$18 \%$ patients with unknown primary) (6). The median survival of subjects with known primary was 9 months, and for subjects with unknown primary, median survival was 6 months, which did not differ for patients with known and unknown primary tumors. Their results also indicated that age, and the absence of other metastases, along with better performance status correlated with improved survival rates. D'Ambrosio and Agazzi also supported that survival was not related to the known and unknown location of the primary tumor (4.5 months for diagnosed and 6 months for undiagnosed primary cancer). For a study that enrolled 342 patients, the authors reported that age ( $<65$ years), treatment status at discharge, asymptomatic brain metastasis, and isolated brain metastasis improved survival (3). Bartelt and Ruttelbach evaluated 916 patients with brain metastases. Among those, 47 patients displayed unknown primary at diagnosis. The authors reported a 4.8 month median survival time for BUMP cancer and also showed that KPS status and resection surgery status were independent prognostic factors of survival (1).

The limitations of this study are that it is a retrospective design; data acquired were from a single-center and relatively small sample size due to the scarcity of BUMP cancer patients. Thus, prospective observations without selection biases and randomized controlled trials are needed to individualize the treatment options, especially with end-stage cancer patients diagnosed with cerebral metastases to improve survival.

\section{CONCLUSION}

Patients with metastatic cerebral tumors at the time of cancer diagnosis have approximately 18.7 months of survival. This study showed that KPS and the number of lesions affected survival outcomes. However, treatment response and survival outcomes substantially depend on patients' overall status and aggressiveness of the systemic disease.

\section{- REFERENCES}

1. Bartelt S, Lutterbach J: Brain metastases in patients with cancer of unknown primary. J Neurooncol 64:249-253, 2003

2. Bertolini F, Spallanzani A, Fontana A, Depenni R, Luppi G: Brain metastases: An overview. CNS Oncol 4:37-46, 2015

3. D'Ambrosio AL, Agazzi S: Prognosis in patients presenting with brain metastasis from an undiagnosed primary tumor. Neurosurg Focus 22(3):E7, 2007 
4. Deshpande K, Buchanan I, Martirosian V, Neman J: Clinical perspectives in brain metastasis. Cold Spring Harb Perspect Med 10(6):a037051, 2020

5. Gaspar L, Scott C, Rotman M, Asbell S, Phillips T, Wasserman T, McKenna WG, Byhardt R: Recursive partitioning analysis (RPA) of prognostic factors in three Radiation Therapy Oncology Group (RTOG) brain metastases trials. Int J Radiat Oncol Biol Phys 37:745-751,1997

6. Gough M, Nielsen M, Coulter IC, Holliman D: Survival outcomes following craniotomy for intracranial metastases from an unknown primary. Int J Clin Oncol 25(8):1475-1482, 2020

7. Lagerwaard FJ, Levendag PC, Nowak PJ, Eijkenboom WM, Hanssens PE, Schmitz PI: Identification of prognostic factors in patients with brain metastases: A review of 1292 patients. Int J Radiat Oncol Biol Phys 43:795-803, 1999

8. Nieder C, Spanne O, Mehta MP, Grosu AL, Geinitz H: Presentation, patterns of care, and survival in patients with brain metastases: What has changed in the last 20 years? Cancer 117:2505-2512, 2011
9. Nolan C, Deangelis LM: Overview of metastatic disease of the central nervous system. Handb Clin Neurol 149:3-23, 2018

10. Nussbaum ES, Djalilian HR, Cho KH, Hall WA: Brain metastases. Histology, multiplicity, surgery, and survival. Cancer 78:1781-1788, 1996

11. Rotta JM, Rodrigues DB, Diniz JM, Abreu BM, Kamimura F, Sousa UO, Botelho RV, Oliveira MF: Analysis of survival in patients with brain metastases treated surgically: Impact of age, gender, oncologic status, chemotherapy, radiotherapy, number and localization of lesions, and primary cancer site. Rev Assoc Med Bras 64:717-722, 2018

12. Smedby KE, Brandt L, Bäcklund ML, Blomqvist P: Brain metastases admissions in Sweden between 1987 and 2006. Br J Cancer 101:1919-1924, 2009

13. Sperduto PW, Berkey B, Gaspar LE, Mehta M, Curran W: A new prognostic index and comparison to three other indices for patients with brain metastases: An analysis of 1,960 patients in the RTOG database. Int J Radiat Oncol Biol Phys 70:510-514, 2008 